\title{
Primary de novo intraosseous carcinoma: Report of a new case
}

\author{
Lorena Gallego $^{1}$, Luis Junquera ${ }^{2}$, Pedro Villarreal ${ }^{3}$, Manuel F. Fresno ${ }^{4}$
}

\author{
${ }^{1}$ Resident. Department of Oral and Maxillofacial Surgery. Central University Hospital, Oviedo \\ ${ }^{2}$ Professor of Oral and Maxillofacial Surgery, University of Oviedo. Staff Surgeon. Department of Oral and Maxillofacial Sur- \\ gery. Central University Hospital, Oviedo \\ ${ }^{3}$ Staff Surgeon. Department of Oral and Maxillofacial Surgery. Central University Hospital, Oviedo \\ ${ }^{4}$ Chief. Department of Pathology. Central University Hospital, Oviedo, Spain. Professor of Pathology, University of Oviedo. \\ Spain
}

Correspondence:

School of Dentistry.

University of Oviedo.

Catedrático José Serrano s/n.

33009 Oviedo. Spain.

Junquera@uniovi.es

Received: $10 / 04 / 2009$

Accepted: 02/08/2009

Gallego L, Junquera L, Villarreal P, Fresno MF. Primary de novo intraosseous carcinoma: Report of a new case. Med Oral Patol Oral Cir Bucal. 2010 Jan 1;15 (1):e48-51.

http://www.medicinaoral.com/medoralfree01/v15i1/medoralv15ilp48.pdf

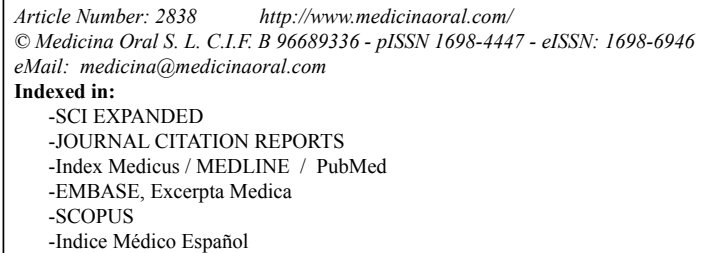

\begin{abstract}
Primary de novo intraosseous carcinoma of the jaws has been rarely reported. We present a new case of this unusual tumour and discuss its histopathological and clinical aspects. The subject was a 76-year-old man who was seen due to complaints of pain and the presence of gingival changes in the left mandible. A panoramic radiograph and computed tomography revealed a large mandibular radiolucency. A segmentary mandibulectomy was performed and histopathologic examination proved that the tumour was an intraosseous squamous cell carcinoma. Surgeons should appreciate the aggressiveness of this tumour, despite adequate surgical treatment.
\end{abstract}

Key words: Intraosseous carcinoma, odontogenic carcinoma, primary intraosseous carcinoma, squamous cell carcinoma.

\section{Introduction}

Primary intraosseous carcinoma (PIOC) of the jaws is a rare tumor presumably developing from residues of the odontogenic epithelium. This tumor was first described by Loos in 1913. The World Health Organization (WHO) in 1972 suggested the term primary intraosseous carcinoma and classified the lesion as an odontogenic carcinoma (1). Later, Waldron and Mustoe's classification was widely accepted and frequently cited (2), which classified such neoplasms as: PIOC ex odontogenic cyst (type 1); presence of malignant ameloblastoma or ameloblastic carcinoma arising de novo (type 2); PIOC arising de novo (type 3 ) and intraosseous mucoepider- moid carcinoma (type 4). Thus, PIOC arising de novo must be considered when no cystic component or other odontogenic tumor cells are demonstrated in the lesion. The etiology is still not clear; probably PIOC derives from the remnants of odontogenic epithelium, epithelial rests of Malassez, or the remnants of the dental lamina (3). Several cases of malignant transformation of odontogenic cysts or odontogenic tumors have appeared in the literature, while primary intraosseous carcinoma arising de novo has been infrequently reported (4-8). We present a case of de novo PIOC affecting the mandible. Differential diagnosis and management considerations of this infrequent tumor are discussed. 


\section{Case report}

A 76-year-old man presented to our hospital complaining of pain and gingival changes in the left mandible. Physical examination showed a non ulcerated gingival hyperplasia adjacent to the left alveolar ridge of the mandible, posterior to the molar area. There was also minor swelling and sensitivity to palpation in the left mandible. Trigeminal nerve function was intact. No cervical lymph node enlargement was noticed. Panoramic radiograph (Fig. 1) and computed tomography (CT) showed an unilocular radiolucency with scalloped borders extending from the ascending ramus to the mandibular first molar (Fig. 2). A biopsy of the le-

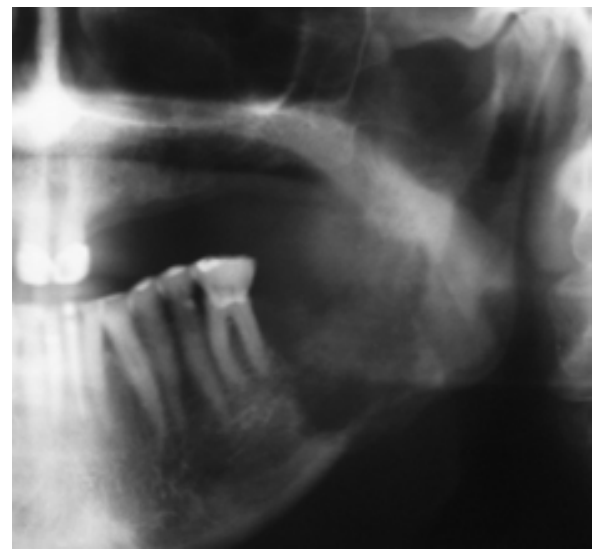

Fig. 1. Panoramic radiograph showing an irregular radiolucent area in the body of the left mandible with diffuse margins.

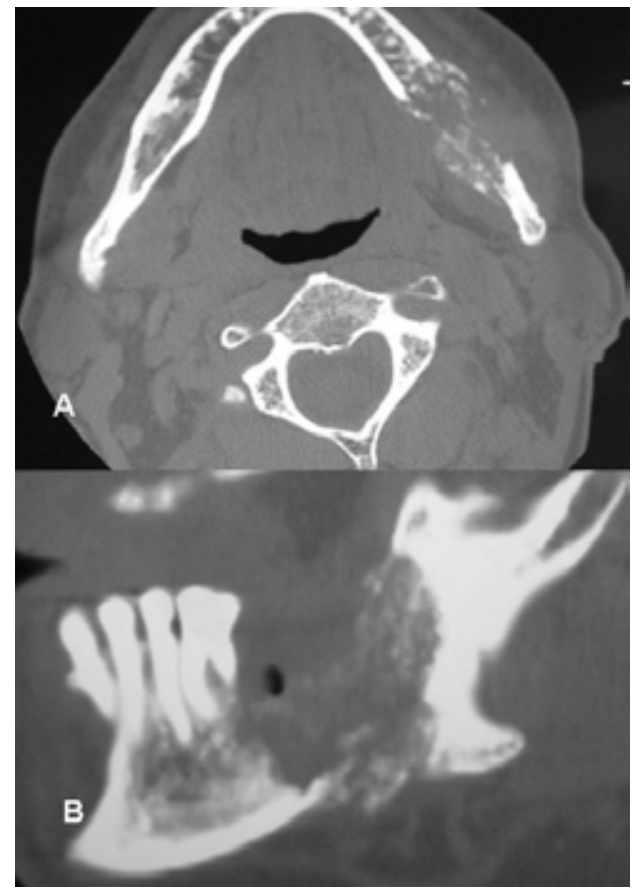

Fig. 2. A.- Axial CT revealed a large oval mass at the left angle of the mandible with extensive bony destruction of the lingual and buccal cortex. B.- Sagital CT image showing extension of tumor from the ascending ramus to the mandibular first molar.

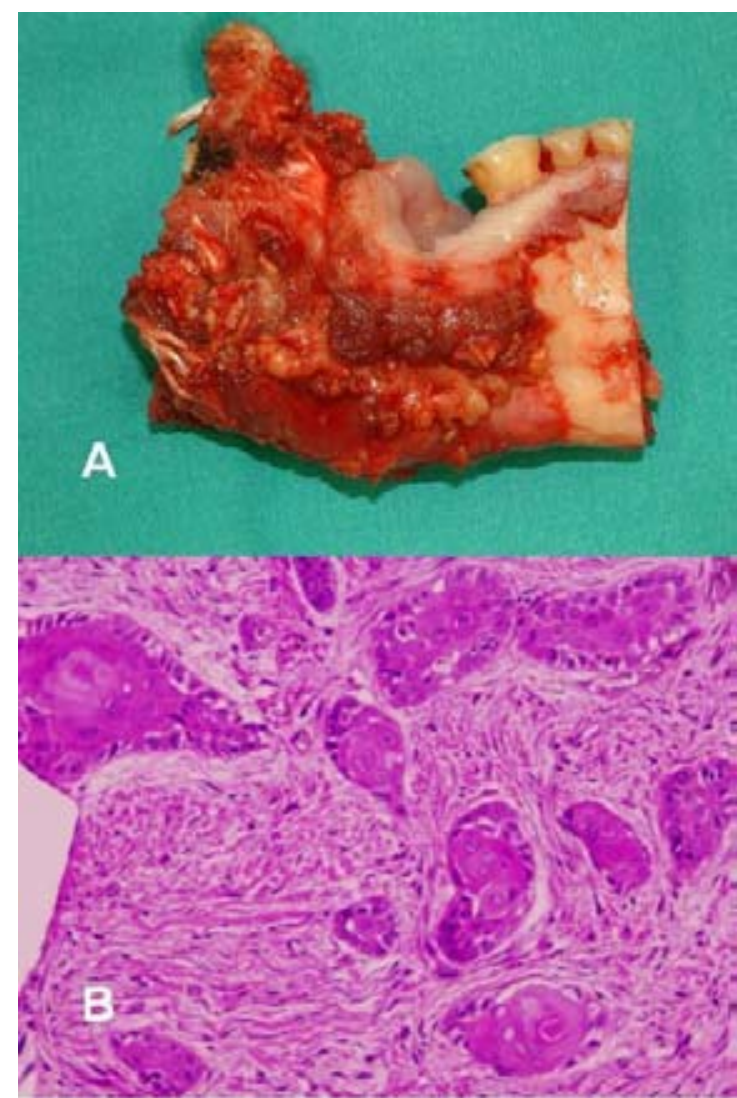

Fig. 3. A.- Gross surgical specimen showing the tumor mass excised with wide margins. B.- Microscopic examination showing infiltrative atypical squamous cell nests (hematoxylin-eosin, x200).

sion was performed and a diagnosis of squamous cell carcinoma was rendered. Two weeks after, a segmentary mandibulectomy with reconstruction using a free peroneal osteocutaneous flap and left supraomohyoid neck dissection were performed under general anesthesia. Intraoperatively, the tumor infiltrated through both the buccal and lingual cortex of the mandible invading the overlying masseter and internal pterygoid muscles (Fig. 3A). The histopathologic examination of the surgical specimen proved that the tumor was an intraosseous carcinoma (Fig. 3B) and no lymphatic metastasis were observed. The patient did not received radiotherapy postoperatively. The 18-months postoperative follow-up revealed neither local nor distant metastasis.

\section{Discussion}

Primary intraosseous carcinoma is an uncommon entity that has been previously well-recognized. The diagnostic criteria proposed for primary intraosseous carcinoma are: absence of initial connection with the overlying mucosa or skin, histologic evidence of squamous cell carcinoma without a cystic component or other odontogenic tumor cells and absence of another primary tumor 
on chest radiographs obtained at the time of diagnosis and during a follow-up period of more than 6 months $(9,10)$. The present case fulfilled these criteria and therefore was classified as de novo PIOC.

De novo PIOC is an epithelial odontogenic malignancy arising from odontogenic epithelial residues in the bone rather than from a pre-existing epithelial lesion. The jaws are the only bones of the skeleton affected primarily by intraosseous carcinoma, which suggests that it originates from epithelial cells present only in them. These epithelial remnants can proliferate and transform into odontogenic carcinoma, a process that could be potentially triggered by an inflammatory process, as a complicated tooth extraction $(11,12)$.

PIOC arising de novo occurs in a wide age group, ranging from 4 to 80 years, with an estimated mean age of 50 years; a male predilection is also noted $(5,7)$. This tumor is most frequently located in the mandible and pain caused by mandibular nerve infiltration is the most common chief complaint of these patients. Paresthesia, accelerated growth with swelling and trismus because of muscle infiltration are also common symptoms. These patients are frequently treated initially for presumed dental problems and this fact often delays the diagnosis of intraosseous carcinomas (4-7).

Radiographically, PIOC shows great variation, but lesions usually show a fully enclosed, irregular pattern of bone destruction with ill-defined margins. Panoramic radiography can be useful in cases of gross disease, but is of limited value in evaluating margins, extension and invasion of the tumor mass. CT allows visualization of the cited features as indicators of aggressiveness and is, therefore, indicated in cases of potentially malignant maxillofacial tumors (13).

The histopathologic features of de novo PIOC are consistent with a diagnosis of squamous cell carcinoma with or without keratinisation. The microscopic differential diagnosis should, therefore, include any lesion that produces squamous epithelium, such as acanthomatous ameloblastoma, squamous odontogenic tumor, metastatic carcinomas and central mucoepidermoid carcinoma. Microscopic examination of serial specimen sections is usually required to rule out the presence of odontogenic cyst lining epithelium or any other odontogenic tumor (14).

Radical surgery with adequate resection appears to be the most important factor in the treatment of this tumor $(4,7)$. In the absence of initial biopsy, the radiolucent lesion may simulate an odontogenic cyst and this situation could lead the surgeon to enucleate the lesion without adequate free margins. Thus, histological diagnosis during the surgical intervention is essential for a correct treatment. Radiotherapy and chemotherapy are used only as palliative therapy or in cases where nerve infiltration is diagnosed (adjuvant therapy) (4). Meta- static spread involving cervical lymph nodes have been described more frequently in PIOC arising de novo than in other types of malignant odontogenic tumors, so prophylactic neck dissection could be recommended $(3,7)$. If there is preoperative evidence of nodal metastasis, involved lymph nodes require block dissection combined with the excision of the primary lesion.

Prognosis is directly related to adequate and complete resection, which obviously requires early detection before the extensive involvement of adjacent tissues or the presence of metastasis to lymph nodes. Thomas et al. (15) reported that overall survival rates of 1,2 , and 3 years were $75.7 \%, 62.1 \%$, and $37.8 \%$, respectively. These findings may advocate for initial aggressive surgical treatment to decrease local recurrence rate.

Additional well-documented examples of PIOC should be reported as it will increase the information about this relatively rare odontogenic neoplasm. With the report of new cases, our experience in its management will increase and more solid theories would be established about the origin and biological behaviour of these truly uncommon neoplasms.

\section{References}

1. Pindborg JJ, Kramer IRH, Torloni H, editors. Histologic typing of odontogenic tumors, jaw cysts and allied lesions. Geneva: World Health Organization; 1972. p. 35-6.

2. Waldron CA, Mustoe TA. Primary intraosseous carcinoma of the mandible with probable origin in an odontogenic cyst. Oral Surg Oral Med Oral Pathol. 1989;67:716-24.

3. Müller S, Waldron CA. Primary intraosseous squamous carcinoma. Report of two cases. Int J Oral Maxillofac Surg. 1991;20:362-5.

4. Lo Muzio L, Mangini F, De Falco V, Pennella A, Farronato G. Primary intraosseous carcinoma of the mandible: a case report. Oral Oncol. 2000;36:305-7.

5. Dimitrakopoulos I, Psomaderis K, Asimaki A, Papaemanouel S, Karakasis D. Primary de novo intraosseous carcinoma: report of two cases. J Oral Maxillofac Surg. 2005;63:1227-30.

6. El-Naaj IA, Krausz AA, Ardekian L, Peled M. Primary intraosseous carcinoma of the anterior maxilla: report of a new case. J Oral Maxillofac Surg. 2005;63:405-9.

7. Chaisuparat R, Coletti D, Kolokythas A, Ord RA, Nikitakis NG. Primary intraosseous odontogenic carcinoma arising in an odontogenic cyst or de novo: a clinicopathologic study of six new cases. Oral Surg Oral Med Oral Pathol Oral Radiol Endod. 2006;101:194-200.

8. González-García R, Sastre-Pérez J, Nam-Cha SH, Muñoz-Guerra MF, Rodríguez-Campo FJ, Naval-Gías L. Primary intraosseous carcinomas of the jaws arising within an odontogenic cyst, ameloblastoma, and de novo: report of new cases with reconstruction considerations. Oral Surg Oral Med Oral Pathol Oral Radiol Endod. 2007; 103:e29-33.

9. Suei Y, Tanimoto K, Taguchi A, Wada T. Primary intraosseous carcinoma: review of the literature and diagnostic criteria. J Oral Maxillofac Surg. 1994;52:580-3.

10. To EH, Brown JS, Avery BS, Ward-Booth RP. Primary intraosseous carcinoma of the jaws. Three new cases and a review of the literature. Br J Oral Maxillofac Surg. 1991;29:19-25.

11. Punnya A, Kumar GS, Rekha K, Vandana R. Primary intraosseous odontogenic carcinoma with osteoid/dentinoid formation. J Oral Pathol Med. 2004;33:121-4.

12. Mosqueda Taylor A, Meneses García A, Ruíz Godoy Rivera LM, Suárez Roa Mde L, Luna Ortiz K. Malignant odontogenic tumors. 
A retrospective and collaborative study of seven cases. Med Oral. 2003;8:110-21.

13. Cavalcanti MG, Veltrini VC, Ruprecht A, Vincent SD, Robinson RA. Squamous-cell carcinoma arising from an odontogenic cyst--the importance of computed tomography in the diagnosis of malignancy. Oral Surg Oral Med Oral Pathol Oral Radiol Endod. 2005;100:365-8.

14. Aboul-hosn Centenero S, Marí-Roig A, Piulachs-Clapera P, Juárez-Escalona I, Monner-Diéguez A, Díaz-Carandell A, et al. Primary intraosseous carcinoma and odontogenic cyst. Three new cases and review of the literature. Med Oral Patol Oral Cir Bucal. 2006;11:E61-5.

15. Thomas G, Pandey M, Mathew A, Abraham EK, Francis A, Somanathan T, et al. Primary intraosseous carcinoma of the jaw: pooled analysis of world literature and report of two new cases. Int J Oral Maxillofac Surg. 2001;30:349-55. 